\title{
An analysis of crystal dissolution fronts in flows through porous media part 2: incompatible boundary conditions
}

\author{
C.J. van Duijn ${ }^{\mathrm{a}}$, P. Knabner ${ }^{\mathrm{b}}$ \& R.J. Schotting ${ }^{\mathrm{c}, *}$ \\ ${ }^{a}$ CWI, P.O. Box 94079, 1090 GB Amsterdam, Netherlands \\ ${ }^{b}$ University of Erlangen-Nürnberg, Institute of Applied Mathematics, Martensstraße 3, D-91058 Erlangen, Germany \\ ${ }^{c}$ Delft University of Technology, Faculty of Civil Engineering and Geosciences, P.O. Box 5048, 2600 GA Delft, The Netherlands
}

(Received 2 July 1996; revised 15 April 1997; accepted 15 September 1997)

\begin{abstract}
A model for transport of solutes in a porous medium participating in a dissolutionprecipitation reaction, in general not in equilibrium, is studied. Ignoring diffusiondispersion the initial value problem for piecewise constant initial states is studied, which e.g. for ionic species include a change of the ionic composition of the solution. The mathematical solution, nearly explicitly found by the method of characteristics up to the (numerical) solution of an integral equation for the position of the dissolution front, exhibits a generalized expanding plateau-structure determined by the dissolution front and the water flow (or salinity) front. (C) 1998 Elsevier Science Limited.
\end{abstract}

Keywords: transport, shock waves, travelling wave, crystal dissolution, porous media, mathematical analysis

AMS classification: $35 \mathrm{R} 35,76 \mathrm{~S} 05$.

\section{INTRODUCTION}

In this paper we continue our study of chemistry affected transport processes in porous media presented in Knabner et al. 7 . The transported solutes are participants in a precipitation-dissolution reaction, which in general is not in equilibrium, but is kinetically controlled. In Ref. ${ }^{7}$ we have set up a model for spatially one-dimensional flow regimes. Throughout Ref. ${ }^{7}$ and the rest of this paper we refer to the solid phase as 'crystal' or 'crystalline solid', which is due to the fact that we have a specific example in mind: the dissolution of a crystalline mineral phase, which occurs as a very thin layer on the grains of the porous medium, see e.g. Willis and Rubin ${ }^{15}$. In fact, the dissolving substance may be either crystalline or amorphous. It was assumed that water content, bulk density, pore velocity $q$ $\left(\mathrm{cm} \mathrm{s}^{-1}\right)$ and diffusion-dispersion coefficient $D\left(\mathrm{~cm}^{2} \mathrm{~s}^{-1}\right)$ are constant. The unknown functions are $u$ and $v$ $\left.(\mathrm{mM} \mathrm{cm})^{-3}\right)$, where $u$ is the molar concentration of one of the reacting participants in solution, $v$ is the scaled concentration of the crystalline solid (both relative to the water volume), and a third unknown $w(-)$, which appears to take into account the nature of the dissolution reaction. For a detailed discussion on the role of $w$ we refer to Section 2 of Ref. ${ }^{7}$. The governing equations are

$$
\begin{aligned}
& \frac{\partial}{\partial t}(u+v)+q \frac{\partial u}{\partial x}-D \frac{\partial^{2} u}{\partial x^{2}}=0 \\
& \frac{\partial v}{\partial t}=k\{g(u ; c)-w K\} \\
& 0 \leq w \leq 1 \text { and } w(x, t)=1 \text { if } v(x, t)>0
\end{aligned}
$$

for $-\infty<x<\infty, t>0$. The positive constants $K$ and $k$ are the saturation constant and a rate parameter, respectively. There is a further function $c$ in the nonlinear function $g$ related to the precipitation reaction. It is a conserved quantity in the sense that it satisfies the linear diffusion-advection equation

$$
\frac{\partial c}{\partial t}+q \frac{\partial c}{\partial x}-D \frac{\partial^{2} c}{\partial x^{2}}=0
$$


for $-\infty<x<\infty, t>0$. The function $c$ is solely determined by the stoichiometry of the precipitation-dissolution reaction. If this is given by

$$
\bar{M}_{12} \leftrightharpoons n M_{1}+m M_{2}
$$

with positive integers $n, m$ where $M_{1}, M_{2}$ denote the species in solution and $\bar{M}_{12}$ the solid, then

$$
c=m c_{1}-n c_{2}
$$

where $c_{1}, c_{2}\left(\mathrm{mM} \mathrm{cm}^{-2}\right)$ are the molar concentrations of $M_{1}, M_{2}$. In eqns (1)-(4), $c_{1}:=u$ is kept as an unknown and $c_{2}$ is substituted by means of ${ }^{6}$. For a spatially independent batch situation the function $\mathrm{c}$ would be constant due to eqn (4), i.e. all possible values of concentrations $c_{1}(t), c_{2}(t)$ lie in an affine subspace of the one-dimensional stoichiometric subspace of the reaction, defined by the condition $c=0$. In the case of ionic species, it is also possible to consider $c$ as the scaled total (positive) electric charge of the solution. This observation helps us in distinguishing two principal situations with respect to a specification by means of initial conditions. We will consider piecewise constant states at $t=0$, i.e.

$$
u(x, 0), v(x, 0), c(x, 0)=0\left\{\begin{array}{lll}
u^{*}, v^{*}, c^{*} & \text { for } & x<0 \\
u_{*}, v_{*}, c_{*} & \text { for } & x>0
\end{array}\right.
$$

We can relate these solutions to solutions of a corresponding boundary value problem for $x>0, t>0$ by considering $u^{*}, v^{*}, c^{*}$ as initial conditions and $u_{*}, v_{*}, c_{*}$ as boundary conditions. Thus there are two situations

$$
c^{*}=c_{*} \text { and therefore } c(x, t)=c=\text { constant }
$$

or

$$
c^{*} \neq c_{*}
$$

In eqn ( 8 ) the boundary (/initial) conditions are compatible in the sense that they belong to the same affine stoichiometric subspace of the reaction or for ionic species that the injected fluid has the same ionic composition as the resident fluid. This situation is the only one which leads to travelling wave solutions being the subject of ${ }^{7}$. Here we concentrate on eqn (9), i.e. on incompatible boundary (/initial) conditions. In this paper we show how to obtain solutions of this problem in the presence of a dissolution front, i.e. a curve in the $(x, t)$-plane separating the region where $v=0$ from the region where $v>0$. To ensure that a dissolution front exists for all $t \geq 0$, one needs

$$
v^{*}=0 \text { and } v_{*}>0
$$

If initially crystalline solid is everywhere present in the flow domain, i.e. $v^{*}>0$ as well, then a dissolution front may occur after a certain finite time interval. Conditions for which this happens are discussed in Section 3.2.

A typical example for the function $g$ is, assuming the thermodynamically ideal mass action law:

$$
g(u ; c)=u^{n}\left(\frac{1}{n}(m u-c)\right)^{m}
$$

where eqn (6) is used. To ensure that $c_{1} \geq 0, c_{2} \geq 0$, only the variable $u \geq(\mathrm{c} / \mathrm{m})_{+}$is allowed, where $a_{+}=a$ for $a \geq u$, $a_{+}=0$ for $a<0$. In general the function $g$ has the following properties to be used later on:

$g(\cdot ; c)$ is strictly monotone increasing for $u \geq(c / m)_{+}$, $g(\cdot ; c)$ is smooth for $u \geq(c / m)+$ (at least Lipschitzcontinuous)

We need the existence of a (unique) $u_{\mathrm{S}}=u_{\mathrm{S}}(c) \geq(\mathrm{c} / \mathrm{m})_{+}$ such that

$$
g(u ; c)=K
$$

i.e. $u_{\mathrm{S}}$ is the solubility for given $c$. Due to the properties of the function $g$ given earlier, the following condition fulfilled by eqn (11) is sufficient for eqn (12):

$$
g\left(\left(\frac{c}{m}\right)_{+} ; c\right)=0
$$

When we require eqn (10), we additionally assume the initial states to be in chemical equilibrium, i.e.

$$
\left(\frac{c}{m}\right)_{+} \leq u^{*} \leq u_{\mathrm{S}}\left(c^{*}\right) \text { and } u_{*}=u_{\mathrm{S}}\left(c_{*}\right) \text {. }
$$

If solid is present everywhere, this would not lead to the appearance of a dissolution front, see eqns (63) and (64). Thus we allow in this case for an initial state for $x<0$ not in equilibrium, which might be thought of as the consequence of an instantaneous removal of saturated fluid. If dispersive transport is negligible compared to advective transport, it is reasonable to let $D \rightarrow 0$, i.e. to cancel the corresponding terms in eqns (1)-(4) and to obtain

$$
\frac{\partial}{\partial t}(u+v)+q \frac{\partial u}{\partial x}=0
$$

for $-\infty<x<\infty, t>0$. The initial value problem eqns (2)-(4), (7) and (15) is known as a Riemann problem. In this paper we consider the analytical and numerical construction of a solution of this Riemann problem. For dominating advective transport, i.e. the limit $D \rightarrow 0$ in eqn (1), we expect a good approximation of the solutions of eqns (1)-(4) and (7) ignoring only certain smoothing effects (see the comparison in Section 5). On the other hand, the treatment of the hyperbolic system by means of the method of characteristics allows a nearly explicit construction of the solution and thus gives detailed information about the qualitative structure of the solution. The function $c$ is found directly, without a priori knowledge about $u, v$ and $w$. It follows from eqns (4) and (7) that for all $t>0$

$$
c(x, t)=\left\{\begin{array}{lll}
c^{*} & \text { for } & x<q t \\
c_{*} & \text { for } & x>q t
\end{array}\right.
$$

To be specific, we assume in the following

$$
c^{*}>c_{*}
$$

The other case can be treated in exactly the same way or transformed to the above situation, which basically corresponds to a renumbering of the dissolved reaction paxticipants. A further property, which holds true for eqn (11), is 
the monotone dependence of the solubility $u_{\mathrm{S}}(\mathrm{c})$ on $c$, i.e. in particular

$$
u_{S}\left(c^{*}\right)>u_{S}\left(c_{*}\right)
$$

In the, analysis we will not make use of this property, but in the figures it is assumed to hold true or implied by the choice of eqn (11). The outline of the paper is as follows. We first construct a solution of the Riemann problem in the case of equilibrium reactions. That is, we take ' $k=\infty$ ' in eqn (2) and replace it by

$$
g(u ; c)=w K
$$

In Section 3.1, we use the method of characteristics, to obtain an explicit representation of the solution, which still is dependent on the dissolution front $x=s(t)$. For this free boundary, which necessarily exhibits a waiting time, we derive an integral equation, which in Section 4 is transformed to a linear Volterra equation of the second kind. This settles the existence of a solution of the integral equation, which then can be used to define a solution of the Riemann problem. In Section 3.2, this procedure is extended to the treatment of $v_{*}, v^{*}>0, u^{*} \leq u_{\mathrm{S}}\left(c^{*}\right)$. In Section 5, an algorithm for the precise approximation of solutions is presented based on the above procedure.

The analysis of multi-component reactive systems with pure advective transport by means of the corresponding hyperbolic systerm has a certain tradition in the chemical engineering literature. Although the situation considered usually allows for more species and reactions then considered here, most of these papers are restricted to the case of equilibrium reactions and a constant number of phases (see e.g. Walsh et al. ${ }^{14}$, Dria et al. ${ }^{3}$, Novak et al. ${ }^{9}$, Bryant et al. ${ }^{2}$, Novak et al. ${ }^{10}$, HelfErich and Klein ${ }^{6}$, Schweich et al. ${ }^{11}$ (and the literature cited therein). If the non-equilibrium case is considered most authors (see e.g. Sevougian et al. ${ }^{12,13}$ ) resort to numerical methods, allowing more complex and realistic situations. The main features of the simple situation studied semi-analytically in this paper are the assumption of non-equilibrium and the appeaxance of a dissolution front.

\section{EQUILIBRIUM}

When studying the transport process at equilibrium we replace the first order eqn (2) by the equilibrium relation (eqn (19)). Thus the equations to be analyzed are eqn (15),

$$
g(u ; c)=w K
$$

subject to eqn (3), where $c$ is given by eqn (16). In this section we construct a solution of this system in the domain $-\infty<x<+\infty$ for $t>0$, which satisfies at $t=0$ the piecewise constant initial distribution (eqn (7)). We recall that the constant states in eqn (7) fulfills eqn (14). To emphasize the role of the dissolution front we impose eqn (10) as well. This simple case is treated for further comparison and introduction of the techniques to be used. In fact the result of this section is well-known in the chemical

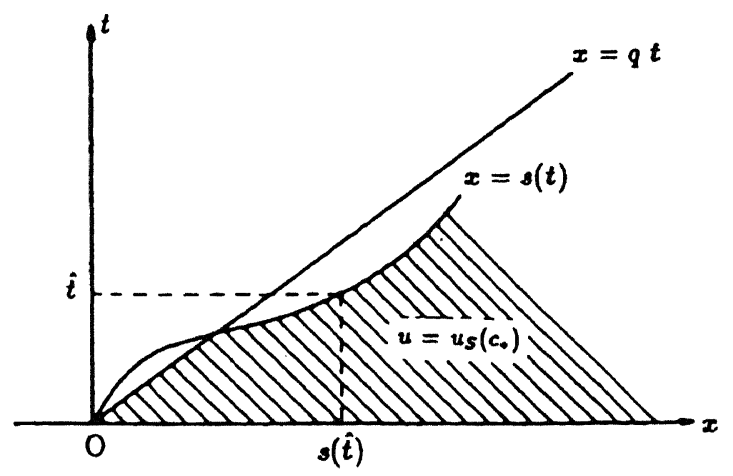

Fig. 1. Dissolution front ahead of the fluid front.

engineering literature (at least formally) and a special case of e.g. Bryant et al. ${ }^{1}$.

For the construction, the following two observations are essential. The first one relates to eqn (20) and says that if $v(x, t)>0$ then $w(x, t)=1$ and by eqn (20)

$$
g(u(x, t) ; c(x, t))=K
$$

In addition, if $x>q t$ then $u(x, t)=u_{S}(c *)=u *$ and if $x<q t$ then $u(x, t)=u_{\mathrm{S}}\left(c^{*}\right)$. The second one is the RankineHugoniot shock condition for solutions of eqn (15). This condition which is based on a mass-conservation argument (see for instance Whitham, ${ }^{16}$ or LeVeque, ${ }^{8}$ ), says that discontinuities or shocks in solutions of eqn (15) propagate with

$$
\text { speed }=\frac{[u]}{[u+v]} q
$$

Here the quantities between the brackets denote the size of the jump discontinuity in $u$ and $v$ across the location of the shock.

Now suppose a dissolution front $x=s(t)$ exists such that

$$
v(x, t)=\left\{\begin{array}{lll}
0 & \text { for } & x<s(t), \\
>0 & \text { for } & x>s(t) .
\end{array}\right.
$$

On physical grounds one expects $s(t) \leq q t$ for all $t>0$, because $q$ denotes the averaged pore velocity of the fluid: i.e. ahead of the front $x=q t$ one expects to find the initial states $u=u *$ and $v=v_{*}$.

The mathematical argument is the following. Suppose the dissolution front moves ahead of the fluid front, as in Fig. 1. Since $w(x, t)=1$ for $x>s(t)$ we find from eqn (21) that $u=$ $u_{\mathrm{S}}\left(c_{*}\right)$ in the shaded region in Fig. 1 . Next select $\hat{t}>0$ such that

$$
s(\hat{t})>q \hat{t} \text { and } \dot{s}(\hat{t})>q
$$

where the dot denotes differentiation. In other words, we have selected a time $\hat{t}$ at which the speed of the dissolution front exceeds $q$. Then by the Rankine-Hugoniot condition (eqn (22)),

$$
u\left(s(\hat{t})^{-}, \hat{t}\right)>u_{*}=u_{S}\left(c_{*}\right)
$$

as $v$ jumps downwards from right to left or does not jump. 


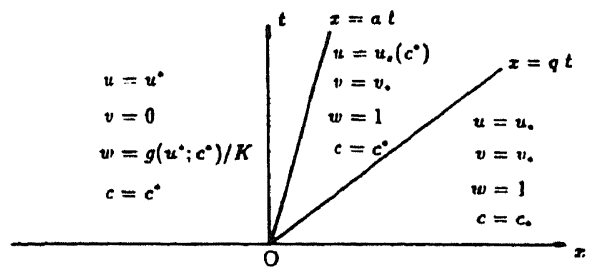

Fig. 2. Level sets of concentrations at equilibrium.

Here $u\left(y^{-}, t\right)=\lim _{x \uparrow y} u(x, t)$ denotes the limit in $y$ from the left and $u\left(y^{+}, t\right)=\lim _{x \downarrow y} u(x, t)$ the limit from the right. But $c(s(\hat{t}), \hat{t})=c *$ and thus by eqns (3) and (20)

$$
u\left(s(\hat{t})^{-}, \hat{t}\right) \leq u_{\mathrm{S}}\left(c_{*}\right)
$$

a contradiction. In other words, eqn (24) implies oversaturation for $u$. But this is not allowed under equilibrium conditions. We further note that if $v$ is discontinuous at the dissolution front, i.e. $v\left(s(t)^{+}, t\right)>0$ then $\dot{\mathrm{s}}(t)=q$ cannot occur. This is a direct consequence of eqn (22). This observation implies that $s(\hat{t})=q \hat{t}$ and $\dot{\mathrm{s}}(\hat{t})=q$ for some $\hat{t}>0$ can also not occur as $v\left(s(\hat{t})^{+}, \hat{t}\right)=v_{*}>0$. Hence

$$
s(t)<q t \text { for all } t>0
$$

The ordering of the fronts and eqns (20) and (21) imply

$$
u(x, t)=\left\{\begin{array}{cc}
\leq u_{S}\left(c^{*}\right) & -\infty<x<s(t) \\
u_{S}\left(c^{*}\right) & s(t)<x<q t \\
u_{S}\left(c_{*}\right) & q t<x<+\infty
\end{array}\right.
$$

Consequently by eqn (22)

$$
0 \leq \dot{s}(t) \leq q \text { for all } t>0
$$

and $\dot{s}(\hat{t})<q$ occurs at points $\hat{t}$ where $v$ is discontinuous. In paxticular this shows that all dissolution fronts are monotone in time. Since $v$ vanishes in the region $x<s(t)$, we have there

$$
\frac{\partial u}{\partial t}+q \frac{\partial u}{\partial x}=0
$$

The initial condition on $u$ for $x<0$, the upperbound in eqns (29) and (30) imply that $u=u^{*}$ for $x<s(t)$. To determine $v$ in the region $x>s(t)$ we use eqns (15) and (28). Combined, they imply that $\partial v(x, t) / \partial t=0$ for $x>s(t), x \neq q t$. Then using the initial condition on $v$ for $x>0$ and the lower bound on $\dot{s}$, we find after integration $v=v_{*}$, for $x>s(t)$. Thus we have constructed a piecewise constant solution of eqns (3), (15) and (20) which satisfies the initial distribution (eqn (7)). The dissolution front follows from the RankineHugoniot condition (eqn (22)): $s(t)=a t$, with

$$
a=\frac{u_{\mathrm{S}}\left(c^{*}\right)-u^{*}}{u_{\mathrm{S}}\left(c^{*}\right)-u^{*}+v_{*}} q(<q)
$$

Across the other shock, $x=q t, v$ is constant. This is consistent with eqn (22). In the chemical engineering literature this front is called the salinity front, see e.g. Bryant et al. ${ }^{1}$ ). In Fig. 2 we show the level set of the solution $\{u, v, w, c\}$ at equilibrium. The separating curves are shock curves at $x=a t$

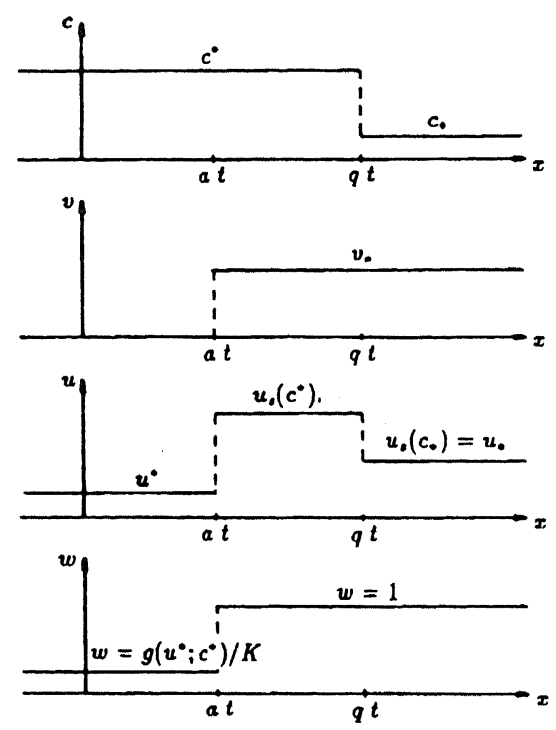

Fig. 3. Sketch of profiles at some $t>0$.

and $x=q t$. Fig. 3 shows a sketch of the profiles of the vaxiables for some $t>0$. A qualitative comparison with the computations of Willis and Rubin ${ }^{15}$ will be given in Section 5 .

One may raise the question if the solution as constructed in this section is the unique solution of the initial value problem. For the following reasons we believe that it is. In the construction, inequalities (eqn (29)) are crucial. They imply directly that the concentrations $u$ and $v$ are constant to the left and the right of a dissolution front, leading to the constant speed eqn (31). In eqn (29) the inequalities are a consequence of the Rankine-Hugoniot condition and the fact that oversaturation is ruled out by requiring $w<1$ in eqn (3). In other words, eqn (29) exhibits local properties of any dissolution front. As outlined above they lead to a piecewise constant solution as presented in this section.

\section{NON-EQUILIBRIUM}

When precipitation-dissolution reactions cannot assumed to be at equilibrium, one needs to incorporate the firstorder reaction eqn (2) in the description. This leads to a much more involved analysis. In this section we construct solutions of the Riemann problem (eqns (2)-(4), (7) and (15)) for two distinct cases. In Section 3.1 we assume eqn (10) to be satisfied, implying that crystalline solid is present only in part of the flow domain, and in Section 3.2 we assume $v^{*}, v_{*}>0$. In this first case a dissolution front exists for all $t \geq 0$. In the second case it may appear in finite time.

\subsection{Crystalline solid partly present}

Inspired by the equilibrium results, i.e. ' $k=\infty$ ', we start with the assumption that a dissolution front exists, as in eqn 


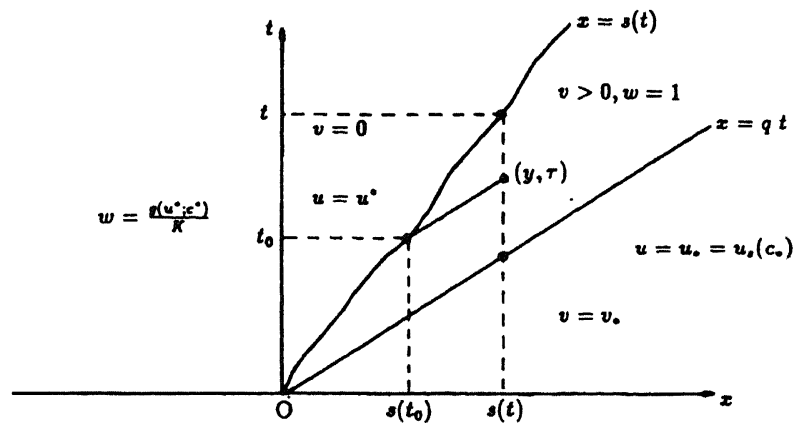

Fig. 4. Dissolution front in the $(x, t)$-plane.

(23), which satisfies inequalities eqn (29). These inequalities are crucial for the construction of a solution. Unfortunately there are no obvious physical or mathematical axguments to support these assumptions. In contrast, the weaker statement $s(t) \leq q t$ for all $t \geq 0$, which is obviously physical, can be justified similarly as in Section 2 . We return to the possibility of existence of solutions not fulfilling these assumptions when discussing the uniqueness at the end of Section 4. The main goal is to derive an equation for the location $x=s(t)$ of the dissolution front.

As in Section 2 we conclude, because of $(\partial v / \partial t)=0$ for $x<s(t)$, that $u=$ const $=\mathrm{u}^{*}$ for $x<s(t)$ and that $w=g\left(u^{*}, c^{*}\right) / K$ there. Similarly for $x>q t$ we have $u=$ const $=u_{*}=u_{\mathrm{S}}\left(c_{*}\right)$ and thus $v=$ const $=v_{*}$. With reference to Fig. 4 , we are going to consider the following problem:

Find $u, v$ and $s$ such that

$$
\begin{aligned}
& \frac{\partial u}{\partial t}+q \frac{\partial u}{\partial x}=k\left\{K-g\left(u ; c^{*}\right)\right\} \\
& \frac{\partial v}{\partial t}=k\left\{g\left(u ; c^{*}\right)-K\right\}
\end{aligned}
$$

for $s(t)<x<q t$ and for $t>0$, subject to

$$
u(s(t), t)=u^{*}, v(s(t), t)=0
$$

and

$$
v(q t, t)=v_{*}
$$

Note that in the composite solution the crystalline concentration $\mathrm{v}$ is continuous across $x=q t$, due to eqn (22) and then eqn (35) holds, while the fluid concentration $u$ possibly has a discontinuity there. Eqn (33) implies continuity of $v$ across the dissolution front $x=s(t)$, which in turn, due to eqn (22), implies continuity of $u$ across $x=s(t)$.

We solve eqns (32) and (33) by the method of characteristics. Choose any point $(y, \tau)$ in the domain $\{(x, t): s(t)<x<$ $q t, t>0\}$, see also Fig. 4. The characteristics of eqn (32) are straight lines in the $(x, t)$-plane, having slope $q$ with respect to the $t$-axis. The characteristic passing through the point $(y, \tau)$, i.e. the curve $x=y+q(t-\tau)$, intersects the dissolution front in the point $\left(s\left(t_{0}\right), t_{0}\right)$, which satisfies

$$
s\left(t_{0}\right)=y+q\left(t_{0}-\tau\right)
$$

Due to $\dot{s}(t) \leq q$ this point is unique and thus is the same for all starting points $(y, \tau)$ satisfying eqn (36). For a given dissolution front $s(t)$, this would determine $t_{0}$ as a function of $y$ and $\tau$, i.e. $t_{0}=t_{0}(y, \tau)$.

Integrating eqn (32) along the characteristic and using eqn (36) yields

$$
\int_{u^{*}}^{u(y, \tau)} \frac{1}{k\left\{K-g\left(z ; c^{*}\right)\right\}} \mathrm{d} z=\tau-t_{0}(y, \tau)=\frac{y-s\left(t_{0}(y, \tau)\right)}{q}
$$

The idea is now to use eqn (33) and the boundary conditions on $v$ to determine the location of the dissolution front, i.e. to find the function $s(t)$. Before we proceed we first introduce for the case $u^{*}<u_{S}\left(c^{*}\right)$ the function $f:[0,+\infty)$ $\rightarrow\left[u^{*}, u_{S}\left(c^{*}\right)\right)$ defined implicitly by the integral

$$
\int_{u^{*}}^{f(\delta)} \frac{1}{k\left\{K-g\left(z ; c^{*}\right)\right\}} \mathrm{d} z=\frac{\delta}{q} \text { for all } \delta \geq 0
$$

and the function $F:[0,+\infty) \rightarrow(0,+\infty)$, defined by

$$
F(\delta)=k\left\{K-g\left(f(\delta) ; c^{*}\right)\right\} \text { for all } \delta \geq 0
$$

Here $\delta$ denotes a distance $y-s\left(t_{0}(y, \tau)\right)$, measured along the characteristic from the dissolution front $x=s\left(t_{0}\right)$ to a point $(y, \tau)$, while $f(\delta)=u(y, \tau)$ denotes the fluid concentration at that point. The fluid concentration $f$ and reaction rate $F$ at any point of a characteristic depend only on this distance $\delta$, which is due to the fact that convection is the only transport mechanism in this single reaction problem. Note that due to the differentiability of $g\left(. ; c^{*}\right)$ at $u=u_{\mathrm{S}}\left(c^{*}\right)$

$$
\int_{u^{*}}^{u} \frac{1}{k\left\{K-g\left(z ; c^{*}\right)\right\}} \mathrm{d} z \rightarrow \infty \text { for } u \rightarrow u_{\mathrm{S}}(c *)
$$

and thus $f$ and $F$ are well defined.

Examples. Let the rate function $g$ be given by the law of mass action (eqn (11)). We can explicitly compute the cases:

$n=1, m=0$ (The linear case, see also Ref. ${ }^{7}$, eqn (40)). Then $g(u ; c)=u$ and $u_{\mathrm{S}}(c)=K$, independent of $c$. We find

$$
f(\delta)=K-\left(K-u^{*}\right) \mathrm{e}^{-\frac{k}{q} \delta}
$$

and

$$
F(\delta)=k\left(K-u^{*}\right) \mathrm{e}^{-\frac{k}{q} \delta}
$$

$n=1, m=1$. (See Fig. 5).

Then $g(u ; c)=u(u-c)$ and

$$
u_{S}(c)=c / 2+(1 / 2) \sqrt{c^{2}+4 K}
$$

We find that $f$ satisfies

$$
\frac{f(\delta)-u_{0}}{u_{\mathrm{S}}-f(\delta)}=\frac{u^{*}-u_{0}}{u_{\mathrm{S}}-u^{*}} e^{\alpha \delta}
$$




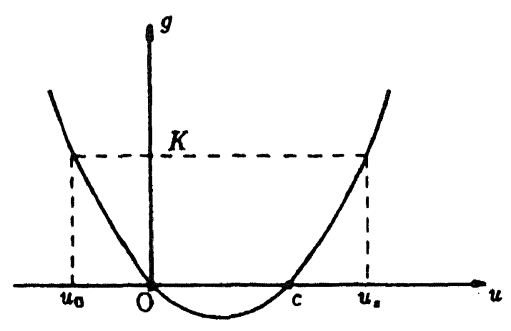

Fig. 5. Rate function $g$ for $n=m=1$. Note that $u^{*} \geq c$.

where

$$
\begin{aligned}
& u_{\mathrm{S}}=u_{\mathrm{S}}(c), u_{0}=u_{0}(c)=\frac{c}{2}-\frac{1}{2} \sqrt{c^{2}+4 K} \\
& \text { and } \alpha=\frac{k}{q}\left(u_{\mathrm{S}}-u_{0}\right)
\end{aligned}
$$

Consequently

$$
F(\delta)=k\left(u_{\mathrm{S}}-u_{0}\right)^{2} \frac{u *-u_{0}}{u_{\mathrm{S}}-u^{*}} \frac{e^{-\alpha \delta}}{\left\{e^{-\alpha \delta}+\frac{u *-u_{0}}{u_{\mathrm{S}}-u^{*}}\right\}^{2}}
$$

When $u^{*}=u_{\mathrm{S}}\left(c^{*}\right)$, which we consider as a degenerate case, we extend the definitions (eqns (38) and (39)) by setting

$$
f(\delta)=u_{S}\left(c^{*}\right) \text { and } F(\delta)=0 \text { for all } \delta \geq 0
$$

Unless stated otherwise we avoid this degeneracy by taking $u^{*}<u_{\mathrm{S}}\left(c^{*}\right)$. This implies $F(\delta)>0$ and $F^{\prime}(\delta)<0$ for all $\delta \geq 0$.

Next we continue the analysis of eqns (32) and (33), by rewriting eqn (37). Take any $t>0$ and let $y=s(t)$. Using eqns (38) and (39) we now write

$$
k\left\{K-g\left(u(s(t), \tau) ; c^{*}\right)\right\}=F\left(s(t)-s\left(t_{0}\right)\right)
$$

for any $s(t) / q<\tau<t$. Here we have used $\dot{s}(t) \geq 0$. In this expression, $t_{0}=t_{0}(s(t), \tau)$ satisfies $t_{0}=t$ when $\tau=t$. Substituting eqn (46) into eqn (33), integrating the result in time and applying the $v$-boundary condition in eqns (34) and (35), yields

$$
\int_{\frac{s(t)}{q}}^{t} F\left(s(t)-s\left(t_{0}\right)\right) \mathrm{d} \tau=v_{*}
$$

Note that in deriving this equation we in particular assumed $s$ to be monotone, but not to be strictly monotone. In the derivation of eqn (47) we can allow for constant parts of $s$, i.e. for times $0 \leq t<t_{2}$ such that $s(t)=s\left(t_{1}\right)$ for all $t_{1} \leq t \leq$ $t_{2}$. In such a case the definition of $t_{0}=t_{0}(s(t), \tau)$ gives for $t$ such that $t_{1} \leq t \leq t_{2}$ :

$$
t_{0}(s(t), \tau)=\tau \text { for } \tau \in\left[t_{1}, t\right]
$$

and the whole derivation of eqn (47) holds true with the following exception: if $s(t)=0$ for $0 \leq t \leq t_{2}$, then the integration leading to eqn (47) cannot be performed for $t \in$ $\left[0, t_{2}\right]$ as $v(0,0)$ is not defined. But on the other hand a

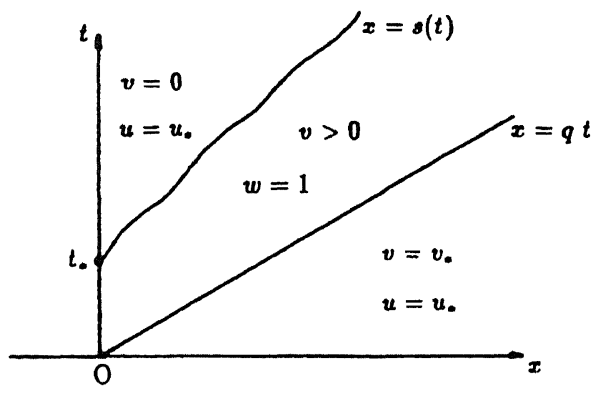

Fig. 6. Waiting time in dissolution front.

dissolution front as sketched in Fig. 4, i.e. $s(0)=0$ and $s(t)>0$ for $t>0$, would lead to a contradiction in eqn (47). Letting $t \downarrow 0$ would make the left hand side zero while $v_{*}>0$ as given. Therefore, we have a waiting time $t_{*}>0$, see Fig. 6 , such that

$$
s(t)=\left\{\begin{array}{rcc}
0 & \text { for } & 0 \leq t \leq t_{*}, \\
>0 & \text { for } & t>t_{*}
\end{array}\right.
$$

From eqn (47) for $t \downarrow t_{*}$, we conclude

$$
t_{*} F(0)=v_{*}
$$

Using eqns (38) and (39) we find for the waiting time the expression

$$
t_{*}=\frac{\nu_{*}}{k\left\{K-g\left(u^{*}, c^{*}\right)\right\}}
$$

Note that in the degenerate case $u^{*}=u_{S}\left(c^{*}\right)$ eqn (51) implies $t_{*}=\infty$. In other words, when $u^{*}$ equals the solubility concentration then the dissolution front remains stagnant. Furthermore, apart from the initial waiting time, no further constant parts of $s$ can occur: if this would be the case, say on the interval $\left[t_{1}, t_{2}\right]$, then we conclude from eqn (47)

$$
\begin{aligned}
& v_{*}=\int_{s\left(t_{2}\right) / q}^{t_{2}} F\left(s\left(t_{2}\right)-s\left(t_{0}\right)\right) \mathrm{d} \tau \\
& =\int_{t_{1}}^{t_{2}} F\left(s\left(t_{1}\right)-s\left(t_{0}\right)\right) \mathrm{d} \tau+\int_{s\left(t_{1}\right) / q}^{t_{1}} F\left(s\left(t_{1}\right)-s\left(t_{0}\right)\right) \mathrm{d} \tau \\
& =\int_{t_{1}}^{t_{2}} F(0) \mathrm{d} \tau+v_{*},
\end{aligned}
$$

which is a contradiction. We want to rewrite the integral in eqn (47) in terms of $t_{0}$ and we do this by using eqn (36). From that equality, with $y=s(t)$, we obtain due to $\dot{\mathrm{s}}(t) \leq q$ a unique correspondence of the points $\tau$ with the points $t_{0}$, where

$$
\tau: \frac{s(t)}{q} \rightarrow t \text { implies } t_{0}: 0 \rightarrow t
$$

and

$$
\frac{\partial \tau}{\partial t_{0}}=1-\frac{1}{q} \dot{s}\left(t_{0}\right)
$$


Applying these observations to eqn (47) yields

$$
\begin{aligned}
& \int_{0}^{t} F\left(s(t)-s\left(t_{0}\right)\right) \mathrm{d} t_{0}=v_{*}+\frac{1}{q} \int_{0}^{t} F\left(s(t)-s\left(t_{0}\right)\right) \dot{s}\left(t_{0}\right) \mathrm{d} t_{0} \\
& =v_{*}+\frac{1}{q} \int_{0}^{s(t)} F(z) \mathrm{d} z
\end{aligned}
$$

where the left-hand side can be slightly rewritten by introducing the waiting time:

$$
\int_{0}^{t} F\left(s(t)-s\left(t_{0}\right)\right) \mathrm{d} t_{0}=t_{*} F(s(t))+\int_{t^{*}}^{t} F\left(s(t)-s\left(t_{0}\right)\right) \mathrm{d} t_{0}
$$

To summarize, we have obtained an integral equation from which the location of the dissolution front can be determined. The precise formulation is: Let $t *$ be given by eqn $(51)$. Then find the function $s(t)$, satisfying eqn (49) and the dissolution front equation (DFE)

$$
(D F E)\left\{\begin{array}{l}
\int_{t_{*}}^{t} F\left(s(t)-s\left(t_{0}\right)\right) \mathrm{d} t_{0}=B(s(t)) \text { for } t \geq t_{*} \text { where } \\
B(s(t)):=t_{*}\{F(0)-F(s(t))\}+\frac{1}{q} \int_{0}^{s(t)} F(z) \mathrm{d} z .
\end{array}\right.
$$

The expression for $B$ follows from eqns (50), (55) and (56). In general we have to rely on numerical methods to solve (DFE). One such method will be discussed in Section 5. Only very special cases can be solved analytically, for instance the case $n=1$ and $m=0$ (the linear case) in the examples, where $F$ is given by eqn (42). For that form of $F$ it is straightforward to solve (DFE) explicitly. The result is

$$
s(t)=\frac{q}{1+k t_{*}}\left(t-t_{*}\right) \text { for } t \geq t_{*}
$$

where

$$
t_{*}=\frac{v_{*}}{k\left(K-u^{*}\right)}
$$

In Section 4 we show how to transform (DFE) into a standard integral equation, from which some characteristic properties of the front can be derived. Having found an expression or approximation for $s(t)$, one has to go back to eqns (37) and (38) to determine $u$. The concentration of the crystalline solid is obtained from integrating eqn (33).

\subsection{Solid present everywhere}

As in the previous case, the concentrations ahead of the fluid front are not affected by the displacing fluid and, therefore, equal to the initial concentrations. Since $v$ is continuous across $x=q t$, the essential parameters which determine the behavior of the concentrations are $u^{*}, v^{*}, c^{*}$ (rather $\left.u_{\mathrm{S}}\left(c^{*}\right)\right)$ and $v_{*}$, see Fig. 7. For definiteness we assume here that $v^{*}<v_{*}$. As long as $v>0$, implying $w=1$, no dissolution front will emerge and we need to solve eqns (32) and

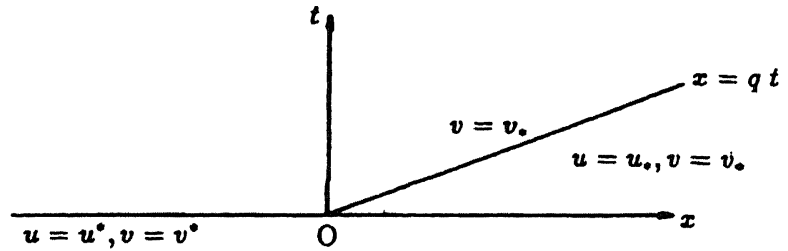

Fig. 7. Initial conditions for the domain $x<q t, t>0$.

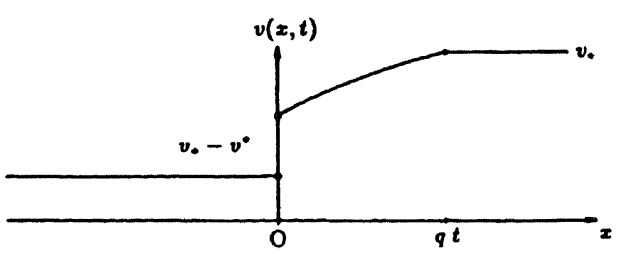

Fig. 8. Crystalline solid distribution for fixed $t>0$.

(33) subject to the conditions shown in Fig. 7. From the physical point of view the initial conditions for $x<0$ are somewhat unrealistic because $u^{*}$ and $v^{*}$ are not in equilibrium. This implies that in a laboratory experiment the resident fluid in the region $x<0$ at $t=0$ has to be instantaneously replaced by fluid with concentration $u^{*}$.The latter is, by approximation, only feasible for (very) small reaction rates.

Integrating the $u$-equation along the characteristic $x-q t=$ constant and the $v$-equation in $t$, we find that

$$
u(x, t)=f(q t) \text { for } x<0 \text { or } 0<x<q t \text { and } t>0
$$

with $f$ defined by eqn (38) and

$$
v(x, t)=\left\{\begin{array}{llc}
v^{*}+u^{*}-f(q t) & \text { for } & x<0, t>0 \\
v_{*}+f(x)-f(q t) & \text { for } & 0<x<q t, t>0
\end{array}\right.
$$

The latter follows immediately from integration of

$$
\frac{\partial v(x, t)}{\partial t}=-\frac{\partial u(x-q t, t)}{\partial t}
$$

in time, see eqn (15). Note that $f$ is monotonically increasing from $f(0)=u^{*}$ towards $f(\infty)=u_{S}\left(c^{*}\right)$. The solid concentration for fixed $t>0$ is sketched in Fig. 8.

Obviously, eqns (60) and (61) are only meaningful if $v>0$. This leads us to consider the following cases.

1. $u^{*}=u_{\mathrm{s}}\left(c^{*}\right)$.

This choice implies $f=$ constant $=u^{*}$, yielding the solution

$$
u(x, t)= \begin{cases}u^{*} & x<q t \\ u_{*} & x>q t\end{cases}
$$

and

$$
v(x, t)= \begin{cases}v^{*} & x<0 \\ v_{*} & x>0\end{cases}
$$




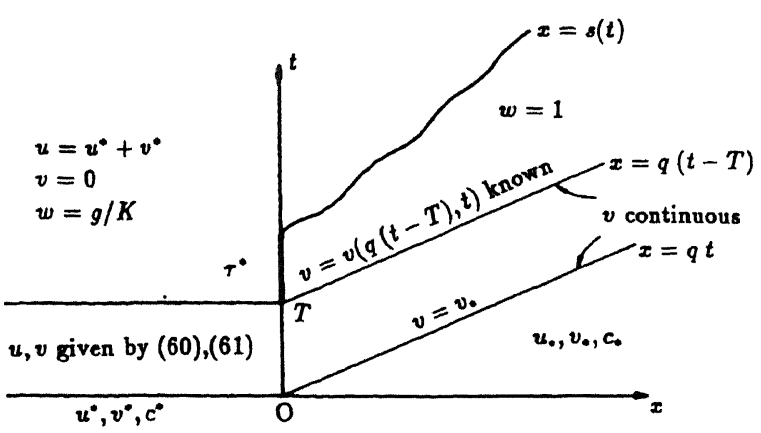

Fig. 9. Concentrations in the $(x, t)$-plane.

2. $u^{*}<u_{S}\left(c^{*}\right)$ and $v^{*} \geq u_{S}\left(c^{*}\right)-u^{*}$.

The second inequality being strict means that the concentration of crystalline solid is too high to be fully dissolved in the fluid. As a consequence eqns (60) and (61) hold for all $t>0$, where

$v(x, t)>v(x, \infty)=\left\{\begin{array}{lc}v^{*}+u^{*}-u_{\mathrm{S}}\left(c^{*}\right) \geq 0, & x<0 \\ v_{*}+f(x)-u_{\mathrm{S}}\left(c^{*}\right)>0, & 0<x<q t\end{array}\right.$

3. $u^{*}<u_{\mathrm{S}}\left(c^{*}\right)$ and $v^{*}<u_{\mathrm{S}}\left(c^{*}\right)-u^{*}$.

Now the second inequality and eqn (61) imply that there exists a finite time $T>0$, defined by

$f(q T)=v^{*}+u^{*}$

such that $v(x, t)>0$ for $0 \leq t<T$ and

$$
v(x, T)=\left\{\begin{array}{lc}
0, & x<0 \\
v_{*}+f(x)-f(q T), & 0<x<q T
\end{array}\right.
$$

The distribution of the concentrations in the $(x, t)$-plane is sketched in Fig. 9. At the point $(0, T)$ a dissolution front emerges as explained in Section 3.1, except that the constant $v$ has to be replaced by the known function $v(q(t-T), t)$. Translating the point $(0, T)$ to the origin by setting $x=x, \tau=$ $t-T$, and writing $s=s(\tau)$ (with $s(0)=0, s(\tau) \geq 0$, for $\tau>0$ ) we find the waiting time $\tau *$

$$
\tau_{*} \tilde{F}(0)=v_{*}-v^{*}
$$

and the modified dissolution front equation (MDFE)

$$
(M D F E)\left\{\begin{array}{c}
\int_{\tau_{*}}^{\tau} \tilde{F}\left(s(\tau)-s\left(\tau_{0}\right)\right) \mathrm{d} \tau_{0}=\tilde{B}(s(\tau)) \text { for } \tau \geq \tau_{*}, \\
\text { where } \\
\tilde{B}(s(\tau))=V(s(\tau))+\tau_{*}\{\tilde{F}(0)-\tilde{F}(s(\tau))\} \\
\quad+\frac{1}{q} \int_{0}^{s(\tau)} \tilde{F}(z) \mathrm{d} z
\end{array}\right.
$$

In the expressions, the function differs from the function $F$ used in (DFE): obviously $u^{*}$ has to be substituted by $f(q T)=u^{*}+v^{*}$ in the definition of a function $\tilde{f}$ in eqn (38) and $F$ is given by eqn (39) by substituting $f$ by $\tilde{f}$. Furthermore, the function $V$ in $\tilde{B}$ is related to $v$ along $x=q(t-T)$. It is found to be

$$
V(s(\tau))=v\left(s(\tau), T+\frac{s(\tau)}{q}\right)-\left(v_{*}-v^{*}\right)
$$

or, using eqn (61),

$$
V(s(\tau))=v^{*}+f(s(\tau))-f(q T+s(\tau))
$$

Note that here the original function $f$ according to eqn (38) appears in eqn (71). Clearly $V(0)=0$. Having determined $s(\tau)$ from eqn (68), (MDFE) and eqn (71), one proceeds as before to find $u$ and $v$ in the region $s(\tau)<x<q \tau, \tau>0$.

In principle a discontinuity of $u$ is possible at $x=q(t-T)$. In fact $u$ is continuous there, which can be seen as follows: due to eqn (60) we have $u\left(q(t-T)^{+}, t\right)=f(q t)=f(x+q T)$. On the other hand the definition of $\tilde{f}(x)$ leads to $u\left(q(t-T)^{-}, t\right)=\tilde{f}$. We have, setting $h(z)=1 /\left(k\left\{K-g\left(z ; c^{*}\right)\right\}\right)$ :

$$
\int_{u^{*}}^{\tilde{f}(\delta)} h(z) \mathrm{d} z=\frac{\delta}{q}+\int_{u^{*}}^{u^{*}+v^{*}} h(z) \mathrm{d} z=\frac{1}{q}\left(\delta+f^{-1}\left(u^{*}+v^{*}\right)\right)
$$

as $u^{*}+v^{*}<u_{\mathrm{S}}\left(c^{*}\right)$ and thus is in the range of $f$. Therefore,

$$
\tilde{f}(\delta)=f\left(\delta+f^{-1}\left(u^{*}+v^{*}\right)\right) f(\delta+q T)
$$

due to equation (66),

in particular we have

$$
u\left(q(t-T)^{+}, t\right)=u\left(q(t-T)^{-}, t\right)
$$

The qualitative analysis concerning dissolution fronts, as given in Section 4, is restricted to (DFE) only. This choice implies no loss of generality. All results/properties carry over to the solution of (MDFE). However, when discussing the numerical results, we do present an example in which a concentration distribution as shown in Fig. 9 will arise.

\section{DISSOLUTION FRONT EQUATION}

Before discussing the qualitative behavior of the dissolution front, i.e. the solution of integral equation (DFE), we recall here that this equation was derived by assuming the structural conditions (eqn (29)). These conditions are consistent with the following results.

Property. Let $s:[t * \infty) \rightarrow[0, \infty)$ be differentiable and satisfy (DFE). Then

1. $s\left(t_{*}\right)=0$;

2.

$\dot{s}\left(t_{*}\right)=q /\left(1-\frac{F^{\prime}(0)}{F(0)} t_{*} q\right) \in(0, q)$

3. $0<\dot{s}(t)<q$ for all $t \geq t *$

4. $s(\infty)=\infty$ 
Proof.

(1) By assumption, $s(t) \geq 0$ for all $t \geq t *$ Since $F^{\prime}(\delta)<0$ for all $\delta \geq 0$, with $F(0)=k\left\{K-g\left(u^{*} ; c^{*}\right)\right\}>0$ and $F(\infty)=0$, we observe that the expression $B$ in (DFE) has the property $B(0)=0, B(x)>0$ for $x>0$. Evaluating (DFE) at $t=t_{*}$ yields $B\left(s\left(t_{*}\right)\right)=0$, implying at once $s\left(t_{*}\right)=0$.

(2) Differentiating (DFE) with respect to $t$ and rearranging terms yields

$$
\dot{s}(t)=\frac{F(0)}{\frac{1}{q} F(s(t))-t_{*} F^{\prime}(s(t))-\int_{t_{*}}^{t} F^{\prime}\left(s(t)-s\left(t_{0}\right)\right) \mathrm{d} t_{0}}
$$

for all $t \geq t *$ Taking $t=t *$ in this expression and using (1) gives the desired result $*$.

(3) Because the right hand side in eqn (75) is strictly positive, the lower bound is immediate. The upperbound is more involved. To show it we argue by contradiction. Thus suppose there exists $\tilde{t}>t *$ such that

$$
\dot{s}(t)<q \text { for all } t_{*} \leq t<\tilde{t} \text { and } \dot{s}(\tilde{t})=q \text {. }
$$

Taking $t=\tilde{t}$ in eqn (75) and estimating

$$
\begin{aligned}
& -\int_{t_{*}}^{\tilde{t}} F^{\prime}\left(s(\tilde{t})-s\left(t_{0}\right)\right) \mathrm{d} t_{0} \\
& =-\int_{0}^{s(\tilde{t})} \frac{F^{\prime}(z)}{\dot{s}} \mathrm{~d} z>\frac{1}{q}\{F(0)-F(s(\tilde{t})\}
\end{aligned}
$$

results in

$$
\dot{s}(\tilde{t})<q\left\{1-q t_{*} \frac{F^{\prime}(s(\tilde{t}))}{F(0)}\right\}^{-1}<q
$$

This contradicts eqn (76) and, therefore, $\dot{s}(t)<q$ for all $t \geq t *$.

(4) To prove this we construct a lower bound which becomes unbounded as $t \rightarrow \infty$. Let

$$
C:=\max _{z \geq 0}\left(-F^{\prime}(z)\right)
$$

Using this upper bound in eqn (75) gives for $t \geq t$ *

$$
\dot{s}(t)>\frac{F(0)}{\frac{1}{q} F(s(t))+t_{*} C+\left(t-t_{*}\right) C} \geq \frac{q F(0)}{F(0)+q C t}
$$

and after integration

$$
s(t) \geq \frac{F(0)}{C} \ln \left\{\frac{1+\frac{q C}{F(0)} t}{1+\frac{q C}{F(0)} t_{*}}\right\}
$$

This shows that $s(t)$ becomes unbounded as $t \rightarrow \infty$, see also Fig. 10.

Having determined these a priori properties of the dissolution front, we now turn to the question of existence. To make use of well-known results, we transform the equation to a standard linear integral equation of the second kind for a new unknown function. Due to monotonicity Property (3), by a change of variable (see the line below eqn (88)), we

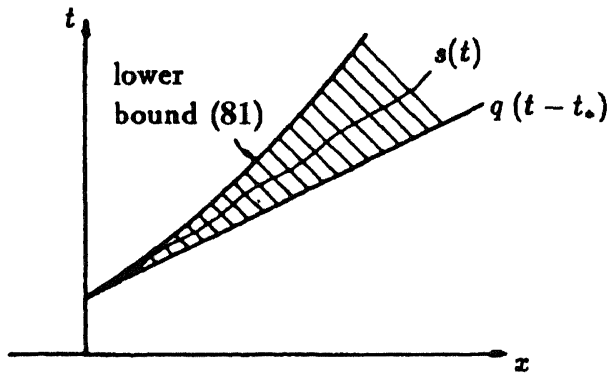

Fig. 10. Estimate of location of dissolution front.

can rewrite (DFE) as a linear integral equation of the first kind for the derivative of the inverse of $s$, denoted by $\phi$. By differentiation with respect to $t$, this equation transforms to

$$
B^{\prime}(x)=F(0) \phi(x)+\int_{0}^{x} F^{\prime}(x-y) \phi(y) \mathrm{d} y \text { for } x \geq 0
$$

where the primes denote differentiation and where $B$ and $F$ are as in (DFE). In particular

$$
B^{\prime}(x)=\frac{1}{q} F(x)-t_{*} F^{\prime}(x)
$$

Eqn (82) has been studied in the mathematics literature and it is known that if $F \in H_{\mathrm{loc}}^{1}\left([0, \infty)\right.$ ) (i.e. $F$ and $F^{\prime}$ are locally square integrable on $[0, \infty)$ ) then eqn (82) has a unique global solution $\phi$ on $[0, \infty)$, see Zabreyko and Mayorova ${ }^{17}$. Furthermore, if $F$ is continuously differentiable (in fact $F$ belongs to $C^{\infty}$ in many relevant examples) then $\phi$ is continuous (or also $C^{\infty}$ ) in $[0, \infty)$. As in the proof of $(3)$, one easily finds

$$
\phi(x)>\frac{1}{q} \text { for all } x \geq 0
$$

Having established the existence of a smooth function $\phi$ satisfying eqns (82) and (84), we are now in the position to define the function $s:[0, \infty) \rightarrow[0, \infty)$ such that $s(t)=0$ for $0 \leq t \leq t *$ and

$$
t-t_{*}=\int_{0}^{s(t)} \phi(x) \mathrm{d} x \text { for all } t>t_{*}
$$

The smoothness of $\phi$ carries over to $s$ in $\left[t_{*}, \infty\right)$ : for instance, if $\phi$ is continuous in $[0, \infty)$ and satisfies eqn $(84)$, then $s$ is continuously differentiable satisfying (3). Thus $s$ is strictly increasing for $t \geq t_{*}$ and also $s(\infty)=\infty$ holds true due to the absence of singularities in $\phi$. In this way, there is a one-toone correspondence of the points $x \geq 0$ and $s(t)$ for $t \geq t$ * To verify that the function $s$ satisfies (DFE), we integrate eqn (82) with respect to $x$ from $x=0$ to $x=s(t)$. This yields for $t \geq t$ *

$$
B(s(t))=F(0)\left(t-t_{*}\right)+\int_{0}^{s(t)}\left\{\int_{0}^{x} F^{\prime}(x-y) \phi(y) \mathrm{d} y\right\} \mathrm{d} x
$$


Writing

$$
\int_{0}^{x} F^{\prime}(x-y) \phi(y) \mathrm{d} y=\frac{\mathrm{d}}{\mathrm{d} x} \int_{0}^{x} F(x-y) \phi(y) \mathrm{d} y-F(0) \phi(x)
$$

gives

$$
B(s(t))=\int_{0}^{s(t)} F(s(t)-y) \phi(y) \mathrm{d} y=\int_{t_{*}}^{t} F\left(s(t)-s\left(t_{0}\right)\right) \mathrm{d} t_{0}
$$

In the last equality we used the variable transformation $s^{-1}(y) \rightarrow t_{0}$, as due to eqn (85) we have $\mathrm{d} / \mathrm{d} y\left(s^{-1}(y)\right)=\phi(y)$. This proves the existence of a continuously differentiable dissolution front for $t \geq t *$ which satisfies (DFE).

We conclude this section with a remark about uniqueness. Any solution of the Riemann problem eqns (2)-(4), (7) and (15), for which a dissolution front $x=s(t)$ according to eqn (23) exists must be of the form discussed in Section 3 with $s$ satisfying (DFE), provided that eqn (29) is satisfied. Now suppose two solutions are possible. They would satisfy Property (3) and thus, using eqn (85), one could define two solutions to the integral eqn (82). But eqn (82) has only one solution which yields a contradiction. The question arises if solutions are possible with dissolution fronts violating eqn (29). If a solution is such that the violation occurs only after some time, i.e. there is a $t_{1}>0$ such that

$$
0 \leq \dot{s}(t) \leq q \text { for } 0 \leq t \leq t_{1} \text { and } \dot{s}\left(t_{1}\right)=0 \text { or } \dot{s}\left(t_{1}\right)=q
$$

then $s$ satisfies the integral equation eqn (47) in the interval $\left[0, t_{1}\right]$ and the waiting time $t *$ according to eqn (51) exists. Assume that $t_{1}>t_{*}$. Then the integral equation (DFE) is valid in $\left[t_{*}, t_{1}\right]$ and Property (3) implies $0<\dot{s}\left(t_{1}\right)<q$, i.e. a contradiction. Thus the only possible further solutions we cannot exclude at the moment have the very unlikely property that there are points $\hat{t}$ arbitrary close to $t=0$ such that $\dot{\mathrm{s}}(\hat{t})<0$ or $\dot{\mathrm{s}}(\hat{t})>q$.

One should bear in mind that not every uniqueness condition is an entropy condition, i.e. a condition which selects from all possible solutions a physical solution. One should also understand that the differential equation describing the dissolution process, has a smoothing effect on the solution. Discontinuities do not occur at the dissolution front, but only at the known front $x=q t$. Therefore, interpreting eqn (22) in terms of 'entropy' is misleading. Van Duijn and Knabner ${ }^{4}$ analyzed in full detail the traveling waves when diffusion is present in the model: the so called viscosity solutions. As solutions are continuous across the dissolution front, no information concerning eqn (22) is to be gained from that analysis.

\section{NUMERICAL METHOD AND RESULTS}

In this section we construct numerical solutions of the Riemann problem eqns (2)-(4), (7) and (15). The numerical solution procedure is based on the method of characteristics
Table 1. Parameters used in the computations.

\begin{tabular}{lll}
\hline$\Theta$ & 0.32 & \\
$\rho_{*}$ & 1.8 & $\left(\mathrm{~g} \mathrm{~cm}^{-3}\right)$ \\
$q^{*}$ & $0.3 \times 10^{-3}$ & $\left(\mathrm{~cm} \mathrm{~s}^{-1}\right)$ \\
$M_{1}$ & $\mathrm{Sr}^{2+}$ & \\
$M_{2}$ & $\mathrm{SO}_{4}^{2-}$ & \\
$n$ & 1 & \\
$m$ & 1 & \\
$c *$ & $2.0 \times 10^{-4}$ & $\left(\mathrm{mmol} \mathrm{cm}^{-3}\right)$ \\
$c *$ & $0.0 \times 10^{-4}$ & $\left(\mathrm{mmol} \mathrm{cm}^{-3}\right)$ \\
$k_{\mathrm{p}}$ & 1 & \\
$k_{\mathrm{d}}$ & $\mathrm{K}$ & $=3.8688 \times 10^{-7}$ \\
$k$ & 0.1 & $\left(1 \mathrm{~m}^{-1}\right)$ \\
$c_{12^{*}}$ & $4.9 \times 10^{-5}$ & $\left(\mathrm{mmol} \mathrm{g}^{-1}\right)$ \\
$c_{12}^{*}$ & $0.0 \times 10^{-5}$ & $\left(\mathrm{mmol} \mathrm{g}^{-1}\right)$ \\
$c_{1 *}$ & $2.0 \times 10^{-4}$ & $\left(\mathrm{mmol} \mathrm{cm}^{-3}\right)$ \\
$c_{1^{*}}$ & $6.22 \times 10^{-4}$ & $\left(\mathrm{mmol} \mathrm{cm}^{-3}\right)$ \\
\hline
\end{tabular}

and follows the lines of Section 3 in detail. We shall give quantitative results for two distinct non-equilibrium cases:

1. The crystalline solid is only present in the flow domain where $x>0$, i.e. $v^{*}=0, v^{*}>0$

2. The crystalline solid is initially present everywhere in the flow domain, i.e. $v^{*}, v_{*}>0$

The parameters used in the computations are adopted from Willis and Rubin ${ }^{15}$ and listed in Table 1 . The differences are the following: $K$ is slightly larger, in Willis and Rubin ${ }^{15}$ only the equilibrium case $k=\infty$ is considered. $K$ is determined by $c_{1^{*}}$ and $c_{2^{*}}$ and thus has to be different from Willis and Rubin ${ }^{15}$, as we do not consider Debye-Hückel corrections in our computations. But note that also these could be handled without problems as the rate function is of general form. The value of fluid concentration $c_{1}^{*}$ used in our computations differs from the value given in the caption of Fig. 3 in Willis and Rubin ${ }^{15}$. The value in the caption is $c_{1}^{*}=\left(\mathrm{Sr}^{2+}\right)_{\mathrm{f}}=2.0 \times 10^{-5} \mathrm{mMol} \mathrm{cm}^{-3}$ (which we used in Ref. ${ }^{7}$ ) while Fig. 3 suggests that the correct value used by Willis and Rubin ${ }^{15}$ equals $c_{1}^{*}=2.0 \times 10^{-4} \mathrm{mMol} \mathrm{cm}^{-3}$. We decided to use the latter value in this paper.

\subsection{Numerical method}

The numerical procedure consists of the following steps: evaluation of integral (eqn (38)) to obtain a numerical approximation of the function $f(\delta)$, substitution of $f(\delta)$ in eqn (39) to obtain $F(\delta)$, numerically solving a Volterra integral equation which follows from (DFE) to find the location of the dissolution front $s(t)$ and finally we go back to eqns (37) and (38) to determine $u$. The concentration of the crystalline solid is obtained by integration of eqn (33).

The integrand of eqn (38) becomes singular when $u_{*}$ tends to the solubility concentration $u_{\mathrm{s}}\left(c_{*}\right)$. This singularity has to be handled with care because we need numerical approximations of $\mathrm{f}(\delta)$ in a wide range of $\delta$ values. We used Clenshaw-Curtis quadrature in combination with symbolic transformation techniques to remove the singularity, as implemented in the computer algebra system Maple, see 


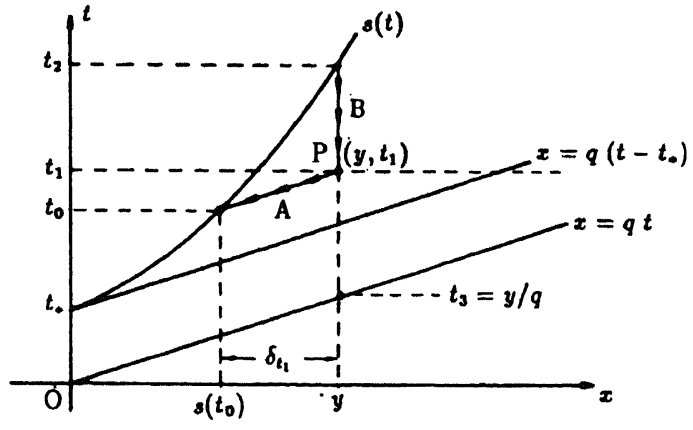

Fig. 11. Construction of solutions in the $(x, t)$-plane.

Geddes ${ }^{5}$. The result of the numerical integration is given as a table $\left.\left[\delta_{i}, f\left(\delta_{i}\right)\right)\right]$, where $\delta_{i}=i \cdot \Delta \delta$. Only in special cases, i.e. $n=1, m=0$ (the linear case) and $n=m=1$, exact evaluation of integral (eqn (38)) is possible. We used the exact eqns (41) and (44) to verify the accuracy of the numerical integration of eqn (38). The discrete result of eqn (38) is used to evaluate eqn (39), i.e. $F\left(\delta_{\mathrm{i}}\right)$ on the $\delta$-grid.

Eqns (82) and (83) can be written as a standard linear Volterra integral equation of the first kind, i.e.

$$
\tau(\delta)=h(\delta)-\int_{0}^{\delta} K(\delta-y) \tau(y) \mathrm{d} y
$$

where

$$
h(\delta)=\frac{1}{q} \frac{F(\delta)}{F(0)}-t_{*} \frac{F^{\prime}(\delta)}{F(0)}
$$

and kernel

$$
K(\delta-y)=\frac{F^{\prime}(\delta-y)}{F(0)}
$$

We solve this equation explicitly, using the trapezoidal rule to discretize the integral in eqn (90). The approximation of the derivatives are chosen central in $\delta$, except in the first integration step where the derivatives are discretized forward in $\delta$. The position of the dissolution front follows from the definition of $r$, hence

$$
t-t_{*}=\int_{0}^{s(t)} \tau(z) \mathrm{d} z
$$

Because $\tau$ is computed at the location of the grid points we have $s(t)=i \Delta \delta$ and the corresponding value of $t$ is found by approximating the right hand side of eqn (93) using Simpson's rule. To compute a profile of the fluid concentration $u$ at a certain time level $t_{1}$ we choose a point $\mathrm{P}\left(y, t_{1}\right)$ in the $(x, t)$-plane (see Fig. 11). We walk backwards along the characteristic through point $P$ and compute the coordinates of the intersection point $\left(s\left(t_{0}\right), t_{0}\right)$ of the characteristic and the free boundary curve $s(t)$. The precise procedure is as follows: start in point $P$, follow the characteristic in the direction of the dissolution front $s(t)$, check in every grid point if the $t$-coordinate of the characteristic is above the corresponding $t$-coordinate of $s(t)$ in that point. If this is the case we use the last and before last step to compute the intersection point of the characteristic and $s(t)$, assuming that the approximation of $s(t)$ is piecewise linear between successive coordinates. This gives the desired value $d_{t_{1}}=y-s\left(t_{0}\right)$, corresponding to point $\mathrm{P}$. Next we use the table of discrete $\left[\delta_{i}, f\left(\delta_{i}\right)\right]$-values to compute the fluid concentration $u\left(y, t_{1}\right)=f\left(\delta_{t_{1}}\right)$ in P. Because $\delta_{t_{i}}$ (usually) does not coincide with one of the $\delta_{i}$-values in the table, we have to interpolate once more. A fluid concentration profile is constructed by repeating this procedure in the region $s\left(t_{1}\right) \leq y \leq q\left(t_{1}-t_{*}\right)$ at a sufficiently large number of points $\mathbf{P}$.

To obtain a numerical approximation of the concentration $\mathrm{v}$ of the crystalline solid in point $\mathrm{P}\left(y, t_{1}\right)$ we first have to obtain values of the fluid concentration in discrete points along the vertical line through $\left(y, t_{2}\right)$ and $\left(y, t_{1}\right)$ using the procedure given above, see line B in Fig. 11. By explicit integration in time of eqn (33) from the position of the free boundary, i.e. $t_{2}$, to the position of P, i.e. $t_{1}$, we obtain $v\left(y, t_{1}\right)$. Full integration from the position of the free boundary $\left(s\left(t_{2}\right), t_{2}\right)$ to the position of the fluid front $\left(y, t_{3}\right)$ has to reproduce the boundary condition $\nu_{*}$ (up to a small error, due to the discrete numerical approximations), which follows from eqn (47). This serves as a check for the accuracy of the numerical procedure. For the linear case $(n=1, m=0)$ we compared results obtained by the numerical procedure and the corresponding exact solutions and found excellent agreement.

\subsection{Results}

In this section we give computed results for the following cases:

1. The linear case $n=1, m=0$ for $v^{*}=0$

2. A non-linear case $n=1, m=1$ for $v^{*}=0$ and for $v^{*}$, $\nu *>0$

3. A non-linear case $n=2, m=2$ for $v^{*}=0$.

Remark. In order to obtain comparable time, space and concentration scales for all cases considered in this section we introduce an artificial factor $a$ that multiplies the function $g$ and assume $K=3.8688 \times 10^{-7}$ to be independent of the values of $n$ and $m$. This implies that only the results of the computations for the case $n=m=1$ have physical meaning. The values of a used in the computations are:

n
1
1
2

$\begin{array}{ll}m & a \\ 0 & 6.22 \times 10^{-4} \\ 1 & 1.0 \\ 2 & 1.83 \times 10^{+6}\end{array}$

5.2.1 The linear case: $n=1, m=0$

Fig. 12 shows the position of the crystal dissolution front in the $(x, t)$-plane for the linear case $(n=1, m=0)$. In this example we have $t_{*}=10499 \mathrm{~s}$. The dissolution front is a straight line which satisfies exactly eqn (58). Due to the introduction of $a$ we have to replace $k t *$ by $a k t_{*}$ in the 


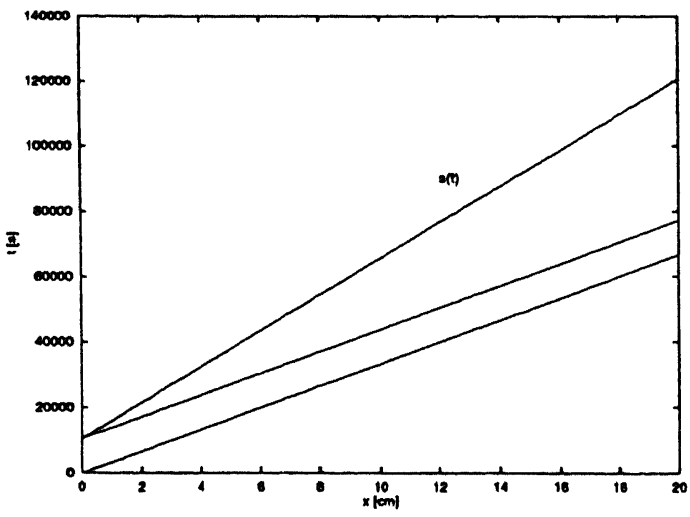

Fig. 12. Dissolution front in the $(x, t)$-plane for the linear case.

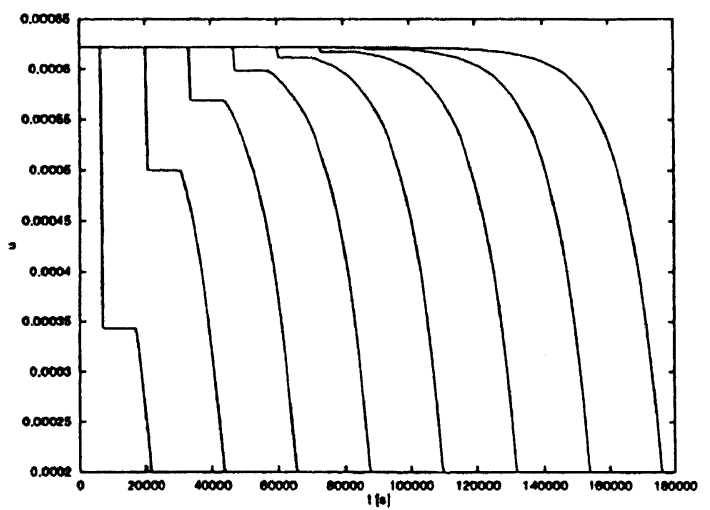

Fig. 13. Breakthrough curves of the fluid concentration $u$ at different positions for the linear case. From left to right the observation points are: $x=2,6,10,14,18,22,26$ and $30 \mathrm{~cm}$ (see Fig. 12).

denominator of eqn (58). Fig. 13 shows breakthrough curves of the fluid concentration at different observation points. An observation independent of this special case is: There are horizontal parts in these curves, which correspond to the fluid concentration in the region in the $(x, t)$-plane where $x_{1} / q \leq t \leq x_{1} / q+t_{*}$ at a given position $x=x_{1}$. In this region $\delta=x_{1}$ is constant and, therefore, $u$ is constant, $u=$ $f\left(x_{1}\right)$ increasing monotonically from $u^{*}$ to $u_{\mathrm{S}}\left(c^{*}\right)$ for $x_{1}$ ranging from 0 to $\infty$. The width of the flat region in all curves is constant and equal to the waiting time $t *$. Fig. 14 shows the time evolution of the crystalline solid concentration at different positions. The regions with constant slope in the $v$-curves correspond to the regions in the breakthrough curves for $u$ where $u$ is constant.

Fig. 15 gives profiles of the fluid concentration at different time levels. We observe several points in the $u$-profiles where the derivative $u_{x}=\partial u / \partial x$ is discontinuous. The discontinuity in $u_{x}$ at the toe of the profiles in Fig. 15 travels with speed $\dot{s}(t)$. A simple computation shows that for the general case

$$
\frac{\partial}{\partial x} u\left(s(t)^{+}, t\right)=k\left\{K-g\left(u^{*} ; c^{*}\right)\right\} \frac{1}{q-s^{\prime}\left(t^{-}\right)}
$$

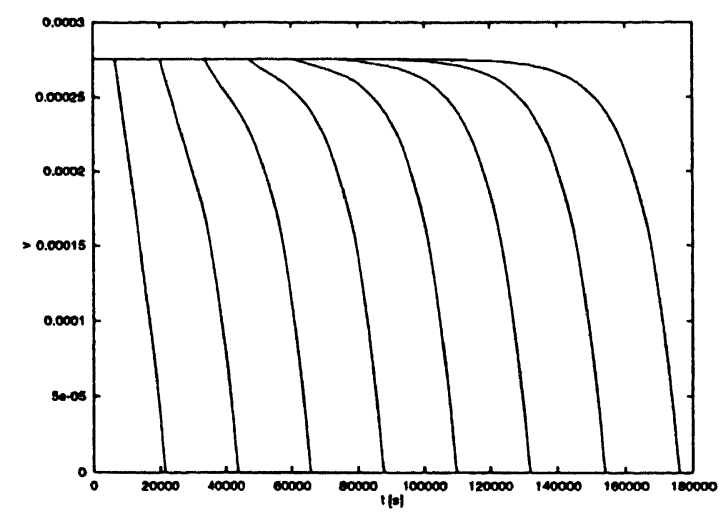

Fig. 14. Time evolution of the crystalline solid concentration $v$ at different positions for the linear case. From left to right the observation points are: $x=2,6,10,14,18,22,26$ and $30 \mathrm{~cm}$ (see Fig. 12)

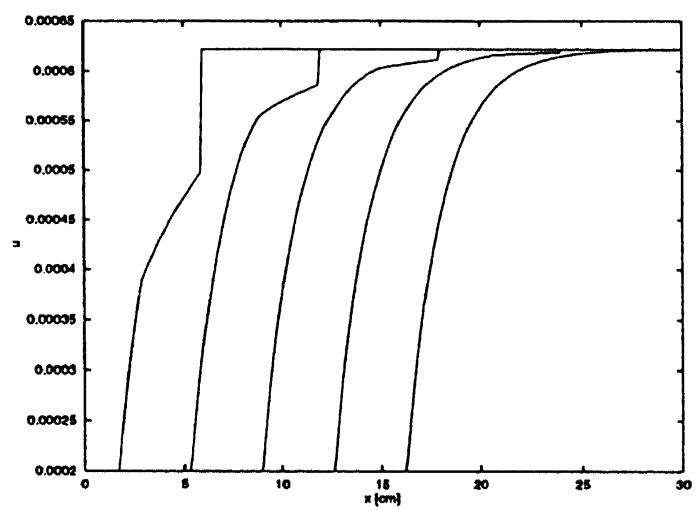

Fig. 15. Fluid concentration profiles at different time levels for the lineax case. From left to right the curves correspond to $t=20000$, $40000,60000,80000,100000$ s (see Fig. 12).

The second discontinuity (from the left) in $u_{x}$ reflects the discontinuity of $\dot{s}(t)$ at $t=t_{*}$. Its position is a point at the line $x=q(t-t *)$ in the $(x, t)$-plane for a given time $t$. In fact, we have in general due to Property (2) for such $(x, t)$

$$
\frac{\partial}{\partial x} u\left(x^{+}, t\right)-\frac{\partial}{\partial x} u\left(x^{-}, t\right)=-f^{\prime}(x) \frac{1}{k t_{*} \frac{\partial}{\partial u} g\left(u^{*} ; c^{*}\right)}
$$

At the fluid front, i.e. the top of the $u$-profile, we have a jump in $u$ which is consistent with the Rankine-Hugoniot shock condition eqn (22), and given by

$$
u\left(q t^{+}, t\right)-u\left(q t^{-}, t\right)=u_{*}-f(q t) \rightarrow u_{*}-u_{\mathrm{S}}\left(c^{*}\right)<0
$$

An exception is the linear case, as here $u_{S}\left(c^{*}\right)=u_{S}\left(c_{*}\right)=$ $u_{*}$ and thus the discontinuity at $x=q t$ vanishes for $x_{1} \rightarrow \infty$. Fig. 16 shows the corresponding profiles of $v$. The derivative $v_{\mathrm{x}}=\partial v / \partial x$ at the dissolution front is discontinuous, due to the discontinuity in $u$ at the front. The constant speed is 


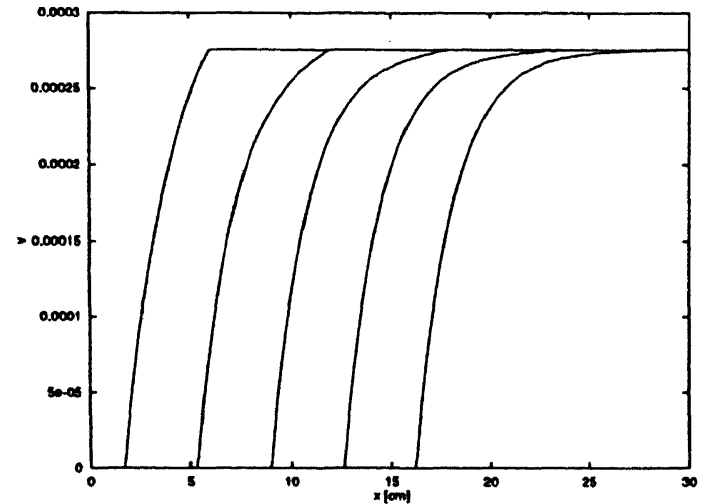

Fig. 16. Crystalline solid concentration profiles at different time levels for the linear case. From left to right the curves correspond to $t=20000,40000,60000,80000,100000 \mathrm{~s}$ (see Fig. 12)

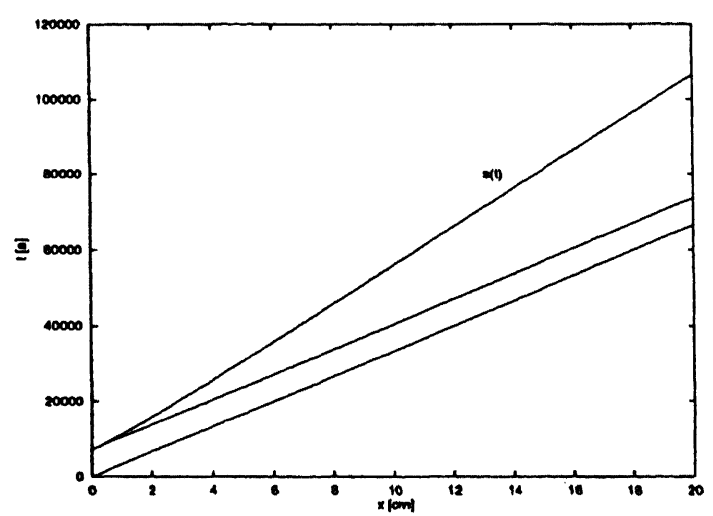

Fig. 17. Dissolution front in the $(x, t)$-plane for the non-linear case $n=m=1$.

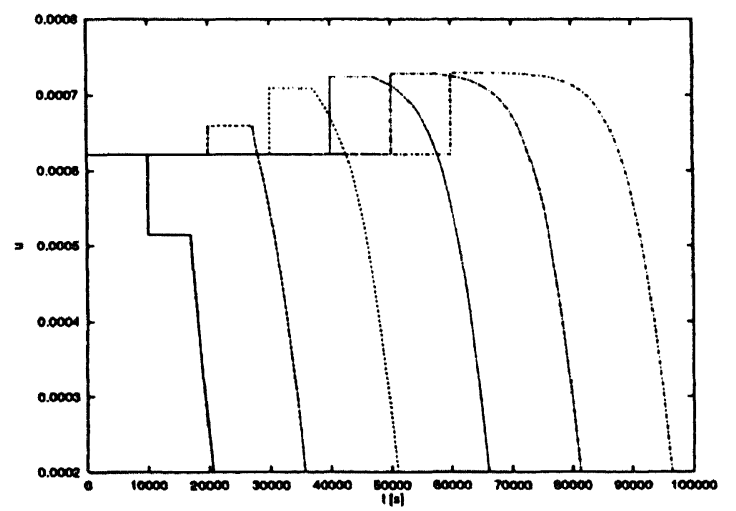

Fig. 18. Breakthrough curves of the fluid concentration at different positions for the non-linear case $n=m=1$. The observation points are $x=3.0,6.0, \ldots, 18.0 \mathrm{~cm}$ (see Fig. 17)

given according to eqn (58) by

$$
\dot{s}(t)=\frac{q}{1+k t_{*}}=\frac{u^{*}-u_{*}}{u^{*}-u_{*}+v^{*}-v_{*}} q
$$

which corresponds to the speed of travelling waves, which exist for constant $c$ [compare Ref. ${ }^{7}$, in particular equation

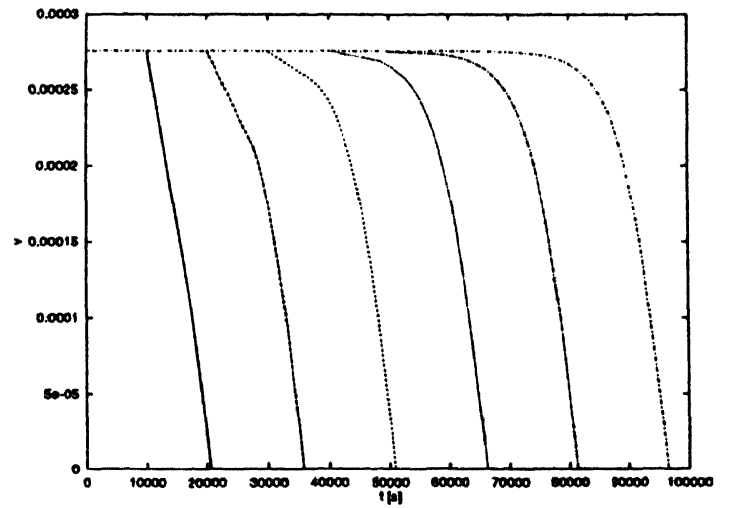

Fig. 19. Time evolution of the crystalline solid concentration at different positions for the non-linear case $n=m=1$. The observation points are $x=3.0,6.0, \ldots, 18.0 \mathrm{~cm}$ (see Fig. 17)

(54)]. By inspection of the explicit solution given by eqns (36), (41), (59) and (97) for the Riemann problem and the explicit solution for the travelling wave problem (note that $g$ is independent of $c$ here, see e.g. the first example in Section 3.1) derived from Ref. ${ }^{7}$, eqns (54) and (93) we see that for taking the shift $L=a t_{*}$, where $a$ is given by eqn (97), the solutions coincide for $x<\left(t-t_{*}\right) q$. In particular there is pointwise convergence in $x$ for $t \rightarrow \infty$ of the solution profile here to the travelling wave solution.

\subsubsection{A non-linear case: $n=1, m=1$}

For this case we shall distinguish between the two sets of initial and boundary conditions as discussed in Sections 3.1 and 3.2 .

\subsubsection{Solid only partly present (See Section 3.1)}

In this case the solubility concentration is given by $u_{\mathrm{S}}(c)=c / 2+1 / 2 \sqrt{c^{2}+4 K}$, i.e. $u_{\mathrm{S}}\left(c^{*}\right)>u_{\mathrm{S}}\left(c_{*}\right)=u_{*}$. We have chosen $c^{*}=u^{*}=2.0 \times 10^{-4}$. Because $c^{*}=0$ we now have $K=u_{*}\left(u_{*}-c_{*}\right)=u_{*}^{2}$, see Table 1 . For the waiting time $t *$ we find $7108.0 \mathrm{~s}$. The dissolution front $s(t)$ is now a curve with slope $\dot{s}<q$ for all $t \geq 0$. Fig. 17 shows the position of the dissolution front in the $(t, x)$-plane. The curve suggests that $\dot{s}\left(t_{*}\right)=q$ which is not true. In fact the $\dot{s}\left(t_{*}\right)$ satisfies Property (2) in Section 4 where in this example it turns out that $F^{\prime}(0) 1 F(0) t * q<1$. Fig. 18 shows breakthrough curves of $u$ for different observation points. The qualitative differences as compared to the linear case are the following: (1) the toe of the $u$-profile does not travel with constant speed but with speed $\dot{s}(t)$. (2) After a certain time the maximum concentration in the profiles exceeds $u_{*}$ and increases in time to the solubility concentration $u_{\mathrm{S}}\left(c^{*}\right)$. (3) The fluid concentration at the fluid front remains discontinuous and jumps either from below or from above to $u_{*}$. Fig. 19 gives the corresponding curves for $v$. The properties of the time evolution of the crystalline solid concentration compare to the those in the linear case, see Figs 14, and 19. Fig. 20 shows the fluid concentration profiles at different 


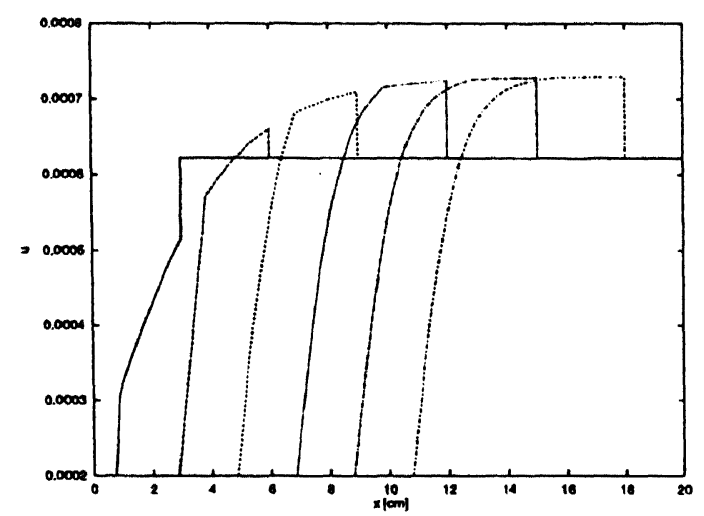

Fig. 20. Fluid concentration profiles at different time levels for the non-linear case $n=m=1$. From left to right the curves correspond to $t=10000.0,20000.0, \ldots, 60000.0 \mathrm{~s}$ (see Fig. 17).

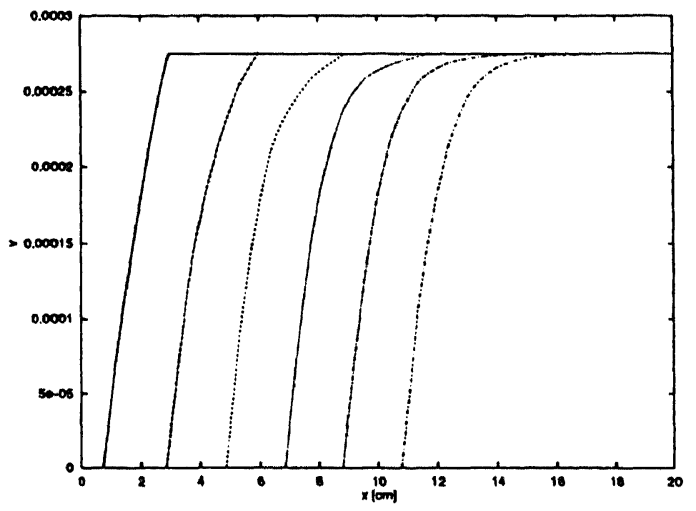

Fig. 21. Crystalline solid concentration profiles at different time levels for the non-linear case $n=m=1$. From left to right the curves correspond to $t=10000.0,20000.0, \ldots, 60000.0$ s (see Fig. 17).

time levels for the non-linear case. The corresponding crystalline solid concentrations are given in Fig. 21.

A quantitative comparison between the solutions of the Riemann problem for the non-linear case and the solutions found by Willis and Rubin ${ }^{15}$ is not possible because they allow for diffusion in their problem and consider only equilibrium reaction. Comparing Fig. 3, Fig. 20 and the corresponding general statements mentioned and Fig. 3 of Willis and Rubin ${ }^{15}$ indicates: the common qualitative property caused by the interplay of transport and dissolution is a 'plateau-structure', which for a fixed time $t$ is defined by the spatial intervals $I_{1}=\{x \mid x<s(t)\}, I_{2}=\{x \mid s(t)<x<q t\}$, $\left.I_{3}=\{x \mid x>q)\right\}$. In $I_{3}$ the solution is given by the 'initial condition' $u_{*}=u_{\mathrm{S}}\left(c_{*}\right)$, in $I_{1}$ by the 'boundary condition' $u^{*}=u_{S}\left(c^{*}\right)$ and in $I_{2}$ at least asymptotically, i.e. for large $t$, by $u_{S}\left(c^{*}\right)$. The piecewise constant structure of Fig. 3 without dispersion and kinetics is smeared out by the addition mechanisms in different ways. Kinetics leads to a smoothing effect in $I_{2}$ such that the transition at $x=s(t)$ becomes continuous and the maximum is attained at $x=q t$. Diffusion

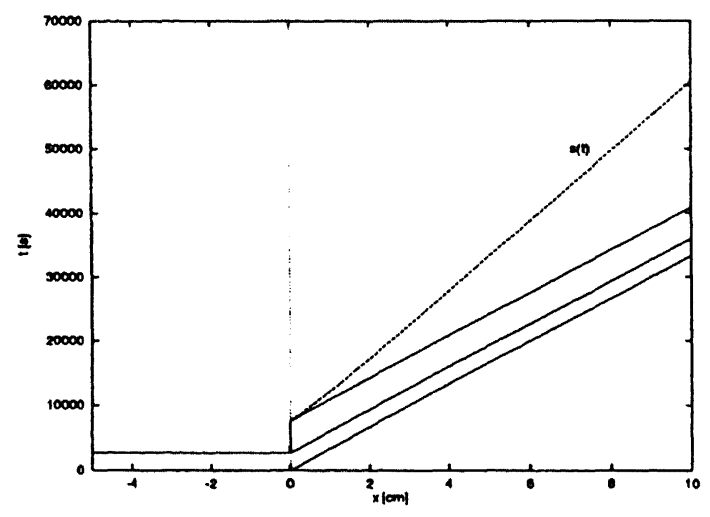

Fig. 22. Dissolution front in the $(x, t)$-plane for the case $n=m=1$ $v^{*}, v_{*}>0$ (compare with Fig. 9).

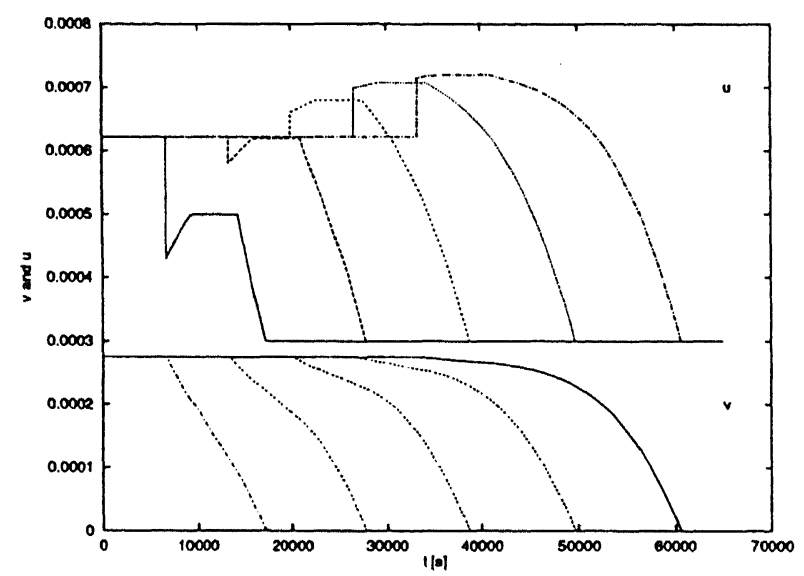

Fig. 23. Breakthrough curves of $u$ (upper curves) and time evolution of $v$ (lower curves) at positions $x=2,4,6,8$ and $10 \mathrm{~cm}$ for the non-linear case $n=m=1, v^{*}, v^{*}>0$.

smooths more, effecting also $I_{1}$ and $I_{3}$ and leading to overall smooth and nonconstant profiles, where the maximum in $I_{2}$ is attained at $x=s(t)$. The solutions for other values of $n$ and $m$ have properties that compare to the solutions of the nonlinear example discussed in this section.

\subsubsection{Crystalline solid present everywhere. (See}

\section{Section 3.2)}

Two characteristic times arise in this case: $T=2680 \mathrm{~s}$, which is the time needed to dissolve all initially present crystalline solid $\left(v^{*}=1.0 \times 10^{-4}\right)$ in the region $x<0$ and $\tau_{*}=4903 \mathrm{~s}$, which is the waiting time for the dissolution front. For $t \geq T$ we have $u=u^{*}+v^{*}=3.0 \times 10^{-4}$ in the region $x<0$. Fig. 22 shows the position of the dissolution front in the $(x, t)$-plane. Fig. 23 gives breakthrough curves of $u$ and the time evolution of $v$ at different positions in one graph. The upper set of curves is $u$ and the lower is $v$. The horizontal parts in the $u$-curves have width $\tau_{*}$ and give rise to linear parts in the $v$-curves, while the increasing parts, which vanish as time proceeds, have width $T$. The 


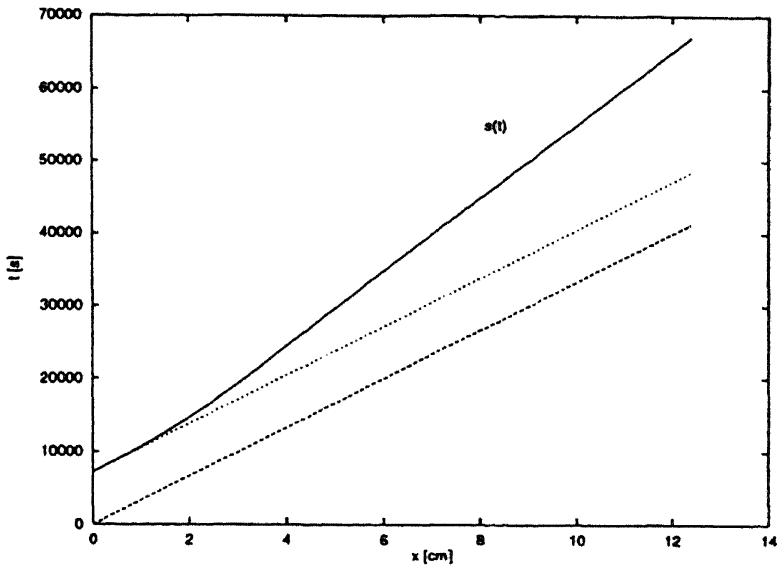

Fig. 24. Dissolution front in the $(x, t)$-plane for the case $n=m=2$,

$$
v^{*}=0
$$

crystalline solid concentration along the line $x=q(t-T)$ in the $(x, t)$-plane is known and given by eqn (61). We used this to check the accuracy of the computations.

\subsubsection{A non-linear case: $n=2, m=2$}

For any combination of $n, m \geq 1$ the function $g\left(u ; c^{*}\right)$ is monotonically increasing and convex in the interval $(\mathrm{c} / \mathrm{m})$ $\leq u \leq u_{S}\left(c^{*}\right)$ and, therefore, we may expect similar qualitative behavior of the solutions. The position of the dissolution front in the $(x, t)$-plane is shown in Fig. 24 . Now the solubility concentration is given by

$$
u_{\mathrm{S}}(c)=\frac{c}{4}+\frac{1}{4} \sqrt{c^{2}+16 \sqrt{K / a}}
$$

The factor $a$ is chosen such that $u_{\mathrm{S}}(c)=7.299 \times 10^{-4}$, as in the case $n=m=1$. The breakthrough curves of $u$ are given in Fig. 25 and the corresponding time evolution of $v$ in Fig. 26.

\section{CONCLUSIONS}

We considered a model for transport and dissolutionprecipitation, where the kinetics of the reaction is taken into account, but diffusion-dispersion is ignored. The appearance and evolution of a dissolution front from corresponding initial states, i.e. the Riemann problem of the hyperbolic system, is investigated. The initial states for the 'charge distribution' $c$ are 'incompatible' in general, i.e. the 'ionic composition' of the fluid changes. The method of characteristics leads to a nearly explicit representation of the solution, where only an implicitly defined function $f$ eqn (38) has to be evaluated numerically and based on $f$ an integral (eqn (57)) has to be solved numerically [or rather the transformed eqns (82) and (83)]. The basic 'plateaustructure' of the solution is revealed being characterized by the dissolution front $x=s(t)$ with speed less than $q$,

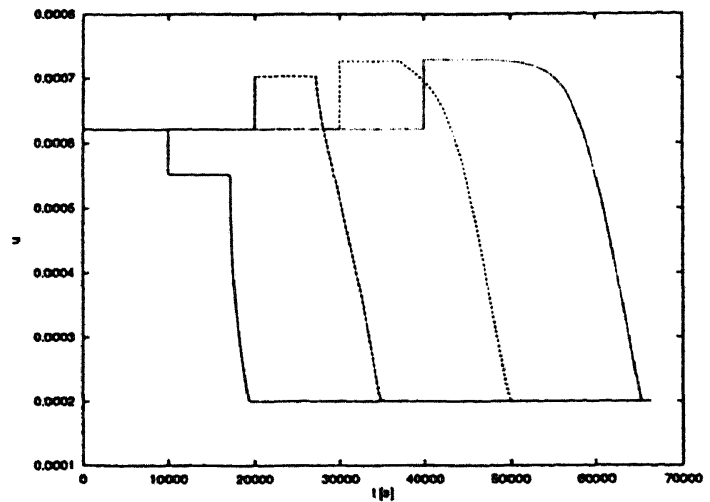

Fig. 25. Breakthrough curves of $u$ at $x=3,6,9$ and $12 \mathrm{~cm}$ for the case $n=m=2, v^{*}=0$.

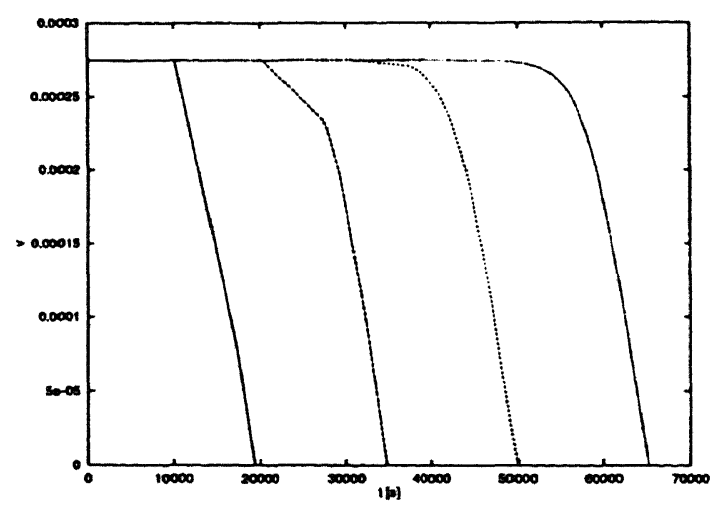

Fig. 26. Time evolution of $v$ at $x=3,6,9$ and $12 \mathrm{~cm}$ for the case $n=m=2, v^{*}=0$.

where (for non-equilibrium) $\partial u / \partial x$ and $\partial v / \partial x$ are discontinuous and the fluid or salinity front $x=q t$, where $u$ and $\partial v / \partial x$ are discontinuous. A comparison of solutions elucidates the role of kinetics and of diffusion-dispersion, which turns out to be similar, but in detail different mechanisms. In addition, due to non-equilibrium, the dissolution front $s$ only starts to move after a positive time $t_{*}$, with positive slope, which implies a discontinuity in $\partial u / \partial x$ at $x=q\left(t-t_{*}\right)$. Because of these properties the solutions are principally different from the travelling wave solutions of Ref. ${ }^{7}$ for 'compatible' boundary conditions and only local convergence can be expected for $t \rightarrow \infty$.

\section{REFERENCES}

1. Bryant, S.L., Schechter, R.S. \& Lake, L.W. Mineral sequences in precipitation-dissolution waves. AIChE J., 1987, 33, 1271-1287.

2. Bryant, S.L., Schechter, R.S. \& Lake, L.W. Interactions of precipitation-dissolution waves and ion exchange in flow through porous media. AIChE J., 1986, 32(5), 751-764.

3. Dria, M.A., Bryant, L., Schechter, R.S. \& Lake, L.W. Interacting precipitation-dissolution waves: the movement of 
inorganic contaminants in groundwater. Water Resour. Res. 1987, 23(11), 2076-2090.

4. Van Duijn, C.J. \& Knabner, P. Travelling wave behaviour of crystal dissolution in porous media flow. Euro. J. Appl. Math., 1997, 8, 49-72.

5. Geddes, K. O., Numerical Integration in a Symbolical Context. In Proceedings of SYMSAC'86, ed. B. Char. ACM Press, New York, 1986, pp. 185-191.

6. Helfferich, F. \& Klein, G., Multicomponent Chromatography. Dekker, New York, 1970.

7. Knabner, P., Van Duijn, C.J. \& Hengst, S. An analysis of crystal dissolution fronts in flows through porous media. Part 1: compatible boundary conditions. Adv. Water Res., 1995, 18(3), $171-185$.

8. LeVeque, R.J., Numerical Methods for Conservation Laws. Birkhäuser, Basel, 1992.

9. Noval, C. F., Lake, L. W. \& Schechter, R. S. Geochemical modelling of two-phase flow with interphase mass transfer. AlChE J., 1991, 37(11), 1625-1633.

10. Novak, C. F., Schechter, R. S. \& Lake, L. W. Diffusion and solid dissolution-precipitation in permeable media. AlChE J., 1989, 35(7), 1057-1072.
11. Schweich, D., Sardin, M. \& Jauzein, M. properties of concentration waves in presence of nonlinear sorption, precipitation-dissolution, and homogeneous reactions I fundamental Wat. Resour. Res., 1993, 29, 723-733.

12. Sevougian, S.D., Lake, L.W. \& Schechter, R.S., KGEOFLOW: A New Reactive Transport Simulator for Sandstone Matrix Acidizing, Society of Petroleum Engineers, Paper (SPE 24780) Februari 1995, pp. 13-19.

13. Sevougian, S.D., Schechter, R.S. \& Lake, L.W. Effect of partial local equilibrium on the propagation of precipitation-dissolution waves. Ind. Eng. Chem. Res., 1993, 32, 2281-2304.

14. Walsch, M.P., Bryant, S.L., Schechter, R.S. \& Lake, W.L., Precipitation and dissolution of solids attending flow through porous media, AIChE J., 30(2) (1984) 317-328.

15. Willis, C. \& Rubin, J. Transport of reacting solutes subject to a moving dissolution boundary: numerical methods and solutions. Water Resour. Res., 1987, 23, 1561-1574.

16. Whitham, G.B., Linear and Nonlinear Waves. Wiley, New York, 1974.

17. Zabreyko, P.P. \& Mayorova, N.L., Integral Equations - A Reference Text. Noordhoff, Leiden, 1975. 